$\Rightarrow$ ACUTE CORONARY SYNDROMES

\section{Exosome-based therapy to repair the injured heart}

Pharmacological and surgical interventions after myocardial infarction (MI) improve clinical outcome but do not restore the function of the damaged tissue. Investigators are therefore exploring new strategies for cardiac repair after MI, including the delivery of stem cells or cardiac muscle cells to the injured heart; however, the cells tend to cause arrhythmias, and their
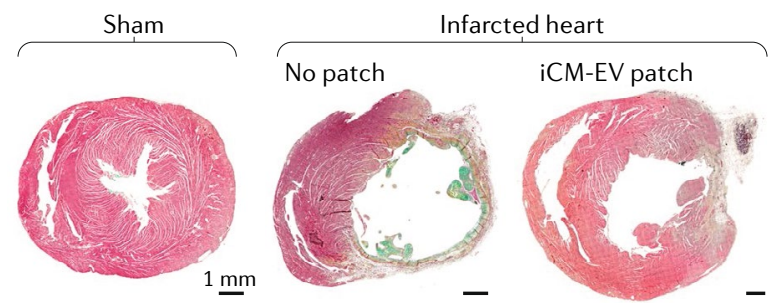

Credit: Reprinted from Liu, B. et al. Cardiac recovery via extended cell-free delivery of extracellular vesicles secreted by cardiomyocytes derived from induced pluripotent stem cells. Nat. Biomed. Eng. https://doi.org/10.1038/s41551-018-0229-7 (2018), Macmillan Publishers Limited effects and mode of action remain unclear. A study describes a new cellfree therapy that improves cardiac recovery after $\mathrm{MI}$ in rats through the delivery of exosomes isolated from cardiomyocytes differentiated from human induced pluripotent stem cells (iPS-CMs).

"We asked ourselves if we could achieve the benefits of the cell therapy of the heart without using the cells," explains lead investigator Gordana Vunjak-Novakovic. Cell transplantations improve cardiac function after MI despite low cell retention in the heart, a 'hit-and-run' mechanism that suggests that the cell secretome mediates cardiac repair. Previous studies have shown that extracellular vesicles (EVs) secreted by stem or progenitor cells can improve cardiac repair; Vunjak-Novakovic and colleagues hypothesized that EVs from iPSCMs (iCM-EVs) would be more therapeutic than EVs from iPS cells (iPS-EVs), given their cardiacspecific components. Bioactive molecules in EVs include microRNAs (miRNAs), and miRNA profiling confirmed that iCM-EVs are enriched in miRNAs that target genes involved in cardiac processes and hypertrophy compared with iPS-EVs.

Human iPS-EVs and iCM-EVs were packaged into hydrogel patches and placed on the myocardium of rats after MI. iCM-EVs reduced cardiac dilatation and improved function compared with iPS-EVs or no treatment at 2 weeks after MI. Additionally, delivery of iCM-EVs but not iPS-EVs significantly reduced infarct size and cardiomyocyte hypertrophy compared with no treatment at 4 weeks after MI. iCM-EVs were also not arrhythmogenic and reduced apoptosis in the injured heart. "We are very excited about the prospects of this treatment. An advantage of this cell-free approach would be the largely simplified FDA regulatory procedure," concludes Vunjak-Novakovic.

Alexandra Le Bras

ORIGINAL ARTICLE Liu, B. et al. Cardiac recovery via extended cell-free delivery of extracellular vesicles secreted by cardiomyocytes derived from induced pluripotent stem cells. Nat. Biomed. Eng. https://doi.org/10.1038/s41551-018-0229-7 (2018)
$\Rightarrow$ HEART FAILURE

\section{Efficacy of cardiac contractility modulation confirmed}

Cardiac contractility modulation (CCM) is safe, improves exercise tolerance and quality of life, and reduces hospitalizations related to heart failure (HF) in patients in NYHA class III-IV and with an ejection fraction (EF) of $25-45 \%$. These findings from the FIX-HF-5C trial, presented at the Heart Rhythm Society Scientific Sessions 2018, confirm previous results from a subgroup analysis of the FIX-HF-5 trial.

CCM therapy is the application of nonexcitatory electrical signals to the heart during the absolute refractory period of the action potential, to augment cardiac muscular contraction without increasing myocardial oxygen consumption. This therapy was developed for the treatment of symptomatic patients with reduced or midrange $E F$ who are not candidates for cardiac resynchronization therapy.
In the FIX-HF-5 trial, CCM treatment effects in primary and secondary end points reached significance only in an exploratory subgroup analysis in patients with an EF of $25-45 \%$. Thus, the FIX-HF- 5 C trial was designed to confirm the safety and efficacy of CCM in this group of patients identified as best responders to therapy. A total of 160 patients with NYHA class III or IV $\mathrm{HF}$ and an EF of $25-45 \%$ were randomly assigned to $\mathrm{CCM}$ plus medical therapy or to medical therapy alone. The investigators used a Bayesian statistical analysis to take advantage of data from the original study.

At 24 weeks, CCM therapy led to improvements in exercise tolerance compared with medical therapy alone, as shown by a difference in peak oxygen consumption of $0.84 \mathrm{ml} \mathrm{O} / \mathrm{kg} / \mathrm{min}$
(95\% Bayesian credible interval: $0.123-1.552 \mathrm{ml} \mathrm{O}_{2} / \mathrm{kg} / \mathrm{min}$ ) between the treatment groups. Quality of life (assessed using the Minnesota Living with Heart Failure Questionnaire), 6-min hall walking test, and NYHA class were also better in patients who received $\mathrm{CCM}$ therapy than in those receiving medical therapy alone. CCM therapy was safe, with $80 \%$ of patients free from device-related adverse events, and use of CCM reduced the composite of cardiovascular death and HF hospitalizations from $10.8 \%$ to $2.9 \%$.

Interestingly, CCM therapy had a stronger effect in each efficacy parameter in patients with an $\mathrm{EF}$ of $35-45 \%$ than in those with an EF of 25-34\%. Future studies with longer follow-up are needed to assess the long-term effects of CCM on mortality and hospitalizations.

Irene Fernández-Ruiz in patients with an EF of $35-45 \%$
ORIGINAL ARTICLE Abraham, W. T. et al. A randomized controlled trial to evaluate the safety and efficacy of cardiac contractility modulation. JACC Heart Fail. https://doi.org/ 10.1016/j.jchf.2018.04.010 (2018) 